

\title{
Effectiveness of the log litaka fibration for 3-folds and 4-folds
}

\author{
Gueorgui Todorov and Chenyang Xu
}

\begin{abstract}
We prove the effectiveness of the log Iitaka fibration in Kodaira codimension two for varieties of dimension $\leq 4$. In particular, we finish the proof of effective log Iitaka fibration in dimension two. Also, we show that for the log Iitaka fibration, if the fiber is of dimension two, the denominator of the moduli part is bounded.
\end{abstract}

\section{Introduction}

One of the main problems in birational algebraic geometry is to understand the structure of pluricanonical maps. If $X$ is a smooth complex projective variety of dimension $n$, then we define the pluricanonical maps

$$
\phi_{r K_{X}}: X \rightarrow \mathbb{P}\left(H^{0}\left(X, \mathrm{O}_{X}\left(r K_{X}\right)\right)\right),
$$

determined by the linear system $\left|r K_{X}\right|$. If this linear system is nonempty for some natural number $r$, which is conjecturally equivalent to $X$ being nonuniruled, then for $r$ sufficiently divisible, the maps $\phi_{r K_{X}}$ become birational to a fixed algebraic fiber space $\phi: X^{\prime} \longrightarrow Y^{\prime}$ called the Iitaka fibration of $X$, and the Kodaira dimension $\kappa(X)$ is set to be $\operatorname{dim} Y^{\prime}$. It is then natural to look for a uniform $r$ (that is, an integer $r$ that depends only on the dimension of $X$ ) for which we are inducing a map birational to the Iitaka fibration. After the monumental work [Birkar et al. 2006] of proving the finite generation of the pluricanonical rings, the effective Iitaka fibration problem is largely related to finding generators which are less than a uniform degree for a given dimension. If $\kappa(X)=\operatorname{dim} X$, then $X$ is called of general type and in this case Hacon- $\mathbf{M}^{c}$ Kernan [2006] and Takayama [2006], following ideas of Tsuji, have shown that for a smooth projective variety of general type and dimension $n$, there exists an integer $r_{n}$, that depends only on $n$, such that $\phi_{r K_{X}}$ is birational for $r \geq r_{n}$. The question remains widely open when the Kodaira dimension is not maximal and it is completely known only up to dimension three [Kawamata 1986; Fujino and Mori 2000; Viehweg and Zhang 2007; Ringler 2007].

MSC2000: primary 14E05; secondary 14J35, $14 \mathrm{~J} 30$.

Keywords: Iitaka fibration, boundedness. 
The current approach for studying this problem is by using Kawamata's canonical bundle formula which identifies the pluricanonical ring of $X$ with the pluricanonical ring of a pair $(Y, \Delta+L)$ of log general type [Fujino and Mori 2000; Prokhorov and Shokurov 2009], where $(Y, \Delta)$ is a KLT pair (KLT = Kawamata $\log$ canonical; see Definition 1.4) and $L$ is a $\mathbb{Q}$-line bundle coming from variation of Hodge structure. This raises the natural question of a log analogue of the above statement. Another setting where boundary divisors naturally occur is in moduli problems. Because of the presence of a boundary divisor the best statement that we can hope for is the following: for $(X, D)$ a KLT pair of Kodaira dimension $\kappa \geq 0$ there is a natural number $m$, that depends only on the coefficients of $\Delta$ and the dimension of $X$ such that $\left|\left\lfloor m\left(K_{X}+\Delta\right)\right\rfloor\right|$ is birational to the Iitaka fibration. To be able to use the canonical bundle formula inductively we have to allow that the coefficients of $\Delta$ lie in a possibly infinite set of rational numbers. The condition that captures the property of this set is the descending chain condition (DCC). At first glance, it may seem to be quite technical and somewhat artificial. However, it turns out to be a very natural and useful condition arising in many questions. See [Shokurov 1992] and [Kollár 1994] for more detailed discussions of this. Thus the general conjecture is formulated as follows.

Conjecture 1.1 (Effective log Iitaka fibration conjecture). Let $(X, D)$ be a $K L T$ (or $\epsilon$-lc) pair such that $K_{X}+D$ is pseudo-effective and the coefficients in $D$ are in a DCC set $A$. Then there is a constant $r$ depending only on the dimension $X$ and on A such that $\left|\left\lfloor r\left(K_{X}+D\right)\right\rfloor\right|$ induces a map birational to the Iitaka fibration.

For a pair $(X, D)$, the log Kodaira dimension is defined as the Iitaka dimension $\kappa\left(K_{X}+D\right)$ [Lazarsfeld 2004, 2.1.3]. For some partial results toward Conjecture 1.1 see [Pacienza 2007]. In this note, we prove the following.

Theorem 1.2. Let $(X, \Delta)$ be a KLT pair of dimension two, three or four and log Kodaira codimension two. Assume that the coefficients of $\triangle$ are in a DCC set of rational numbers $\mathscr{A} \subset[0,1]$. Then there is an explicitly computable constant $m$ depending only on the set $\mathscr{A}$ such that $\left\lfloor m\left(K_{X}+\Delta\right)\right\rfloor$ induces the Iitaka fibration.

The proof of the above theorem contains three parts. First, we prove the dimension-two case of Theorem 1.2. This is done in Theorem 3.1 and the hardest case there is when $X$ itself is a ruled surface over a curve of positive genus. This completes the effectiveness of log litaka fibration in dimension two (for the other cases, see [Todorov 2008]). For this case, it relies on results from [Alexeev 1994] and [Alexeev and Mori 2004].

Then for the higher dimensional case, the key tool is the canonical bundle formula [Kawamata 1998; Fujino and Mori 2000; Kollár 2007]. In our case it roughly says that if $f: X \longrightarrow Y$ is the Iitaka fibration for $K_{X}+\Delta$ and $K_{X}+\Delta=f^{*} D$ for some $\mathbb{Q}$-divisor $D$ on $Y$, then we can define the discriminant or divisorial part 
on $Y$ for $K_{X}+\Delta$ to be the $\mathbb{Q}$-Weil divisor $B_{Y}:=\sum_{P} b_{P} P$, where $1-b_{P}$ is the maximal real number $t$ such that the $\log$ pair $\left(X, \Delta+t f^{*}(P)\right)$ has $\log$ canonical singularities over the generic point of $P$. The sum runs over all codimension-one points of $Y$, but it has finite support. The moduli part or $J$-part is the $\mathbb{Q}$-line bundle $M_{Y}$ on $Y$ that satisfies

$$
K_{X}+\Delta=f^{*}\left(K_{Y}+B_{Y}+M_{Y}\right) .
$$

If $F$ is the general fiber of $f$ and if $h^{0}\left(F, b\left(K_{F}+\Delta_{\mid F}\right)\right) \neq 0$, then for every integer $r$ divisible by $b$ we have

$$
H^{0}\left(X,\left\lfloor r\left(K_{X}+\Delta\right)\right\rfloor\right)=H^{0}\left(Y,\left\lfloor r\left(K_{Y}+B_{Y}+M_{Y}\right)\right\rfloor\right) .
$$

Using ideas of Mori and Fujino, and the two-dimensional case of Theorem 1.2, we can bound the denominators in relative dimension two.

Theorem 1.3. When the relative dimension of $f$ is two, there is a natural number $m$ depending only on the coefficients of $\Delta$ such that $m M$ is Cartier.

Note that Theorem 1.3 holds for pairs of arbitrary dimension.

Finally, after these preparations, to finish the proof it suffices to observe that the results of [Fujino and Mori 2000] in Kodaira dimension-one case and of [Viehweg and Zhang 2007] in Kodaira dimension-two case can be indeed generalized to a log version. Namely, for an $n$-dimensional pair $(X, \Delta)$ of $\log$ Kodaira dimension one or two with Iitaka fibration $f: X \rightarrow Y$ with general fiber $F$, there is a constant $m$, that depends only on the number $b$ satisfying $b\left(K_{F}+\Delta_{\mid F}\right) \sim 0$, the middle Betti number of the associated $b$-fold cyclic cover and the coefficients of $\Delta$, such that $\left\lfloor m\left(K_{X}+\right.\right.$ $\Delta)\rfloor$ induces the Iitaka fibration. In the three-dimensional case of Theorem 1.2, the base is a curve and the conclusions follow from Theorem 1.3 by the same arguments as in [Fujino and Mori 2000]. When the dimension is four, a key observation is that many results in [Alexeev and Mori 2004] can be improved by adding a nef divisor (Proposition 4.3). Similar statements were essentially proved in [Viehweg and Zhang 2007], but we will simplify the proof by putting it in the context of [Alexeev 1994; Alexeev and Mori 2004] and the recent mainstream of investigations on adjoint linear systems.

We remark that Conjecture 7.13 of [Prokhorov and Shokurov 2009] — in fact a list of conjectures - concerns the solution of the effective Iitaka fibration problem. We have shown item (2) of that list, in the relative dimension-two case. (However, we do not prove the semiampleness statements in items (1) and (3) of Prokhorov and Shorukov's Conjecture 7.13.)

The paper is structured as following. In Section 2, we show that the DCC assumption on the coefficients of the boundary indeed forces that the KLT surface pairs of $\log$ Kodaira dimension zero to be $\epsilon$-log canonical, for some $\epsilon$ depending 
on the set of coefficients. In Section 3, we prove that under the same assumption there is a uniform $b$ such that $b\left(K_{S}+B\right) \sim 0$. In Section 4, using ideas of Mori and Fujino, we deduce Theorem 1.3 and complete the proof of Theorem 1.2.

Notations and conventions. We will work over the field of complex numbers $\mathbb{C}$. A $\mathbb{Q}$-Cartier divisor $D$ is nef if $D \cdot C \geq 0$ for any curve $C$ on $X$. We call two $\mathbb{Q}$ divisors $D_{1}, D_{2} \mathbb{Q}$-linearly equivalent $D_{1} \sim_{\mathbb{Q}} D_{2}$ if there exists an integer $m>0$ such that $m D_{i}$ are integral and linearly equivalent. We call two $\mathbb{Q}$-Cartier divisors $D_{1}, D_{2}$ numerically equivalent $D_{1} \equiv D_{2}$ if $\left(D_{1}-D_{2}\right) \cdot C=0$ for any curve $C$ on $X$. A $\log$ pair $(X, \Delta)$ is a normal variety $X$ and an effective $\mathbb{Q}$-Weil divisor $\Delta$ such that $K_{X}+\Delta$ is $\mathbb{Q}$-Cartier. A projective morphism $\mu: Y \longrightarrow X$ is a log resolution of the pair $(X, \Delta)$ if $Y$ is smooth and $\mu^{-1}(\Delta) \cup$ exceptional set of $\mu$ \} is a divisor with simple normal crossing support. For such $\mu$ we write $\mu^{*}\left(K_{X}+\Delta\right)=K_{Y}+\Gamma$, and $\Gamma=\Sigma a_{i} \Gamma_{i}$ where $\Gamma_{i}$ are distinct integral divisors.

Definition 1.4. A pair is called KLT (resp. lc, $\epsilon$-lc) if there is a log resolution $\mu: Y \longrightarrow X$ such that in the above notation we have $a_{i}<1$ (resp. $a_{i} \leq 1, a_{i} \leq 1-\epsilon$ ). The number $1-a_{i}$ is called the log discrepancy of $\Gamma_{i}$ with respect to the pair $(X, \Delta)$.

For conventions and results about DCC set we refer to [Alexeev and Mori 2004], in particular, 2.2-2.7 and 3.4-3.6. When we say some quantity is bounded, it always means there is a computable bound depending on the data we give. We will not keep track of the explicit bound, but it does not require much effort to do so, following the arguments in [Alexeev and Mori 2004]. (Most of the results about surfaces that we use appear already in [Alexeev 1994], but to make the constants explicitly computable, we refer to the joint paper, which is more recent.)

\section{2. $\epsilon$ - $\log$ canonicity}

The main result of this section is this:

Theorem 2.1. Let $\left(S, B=\sum b_{i} B_{i}\right)$ be a KLT projective surface pair. If $K_{S}+B \equiv 0$ and the coefficients of $B$ are in a DCC set $\mathscr{A}$, then there exists an $\epsilon=\epsilon(\mathscr{A})>0$ that only depends on $\mathscr{A}$ such that $(S, B)$ is $\epsilon$-log canonical.

Proof. We can run a minimal model program for $S$,

$$
S=S_{0} \rightarrow S_{1} \rightarrow \cdots \rightarrow S_{n}
$$

By pushing forward $B$ to $S_{n}$, we see that it suffices to prove the statement for $S_{n}$. So we need only prove the case that $S$ is a $\log$ del Pezzo surface of Picard number $1, S$ admits a Fano contraction to some curve with a general fiber $\mathbb{P}^{1}$, or $S$ has $K_{S} \equiv 0$. This is done in Lemmas 2.4-2.6.

The main tool we use is the following theorem, 
Theorem 2.2 [Alexeev and Mori 2004, Theorem 3.2]. Let $\mathscr{A} \subset[0,1]$ be an arbitrary DCC set. There exists a constant $\delta=\beta(\mathscr{A})$ depending only on $\mathscr{A}$ such that the following holds. Let $X$ be a normal projective surface, $B_{j}$ be divisors on $X$, and let $b_{j}, x_{j}$ be positive real numbers. Assume that

(1) $X$ is a singular $\mathbb{Q}$-factorial surface with $-K_{X}$ ample and $\rho(X)=1$;

(2) $b_{j}>0$ and $b_{j} \in \mathscr{A}$;

(3) $1-\delta<x_{j} \leq 1$;

(4) at least one $x_{j}$ is strictly less than 1 ;

(5) the pair $\left(X, \sum x_{j} b_{j} B_{j}\right)$ is $l c$.

Then the divisor $K_{X}+\sum x_{j} b_{j} B_{j}$ is not numerical equivalent to 0 .

Lemma 2.3 [Alexeev and Mori 2004, Lemma 3.6]. Let A be a DCC set containing 1. Let $a, \delta>0$ such that $\delta \leq m f_{2}(\mathscr{A}, a)$ (see [Alexeev and Mori 2004, 3.5] for the definition of the function $m f_{2}(\mathscr{A}, a)$ ). Consider finitely many $b_{j}, x_{j} \in R$ such that $0<b_{j} \in \mathcal{A}, 1-\delta<x_{j} \leq 1$ for all $j$, and $x_{j}<1$ for some $j$. Then $\sum x_{j} b_{j} \neq a$.

Lemma 2.4. If $S$ is a log del Pezzo surface of Picard number $1 . B=\sum b_{j} B_{j}$ is a $\mathbb{Q}$-divisor such that $K_{S}+B \equiv 0,(S, B)$ is KLT and the coefficients of $B$ are in a $D C C$ set $\mathscr{A}$. Then $S$ is $\epsilon=\epsilon(\mathscr{A})$-log canonical.

Proof. We assume $1 \in \mathscr{A}$. If $(S, B)$ is not $\epsilon$ - $\log$ canonical, then we can extract a divisor $f: S^{\prime} \rightarrow S$ such that

$$
f^{*}\left(K_{S}+\sum b_{j} B_{j}\right) \equiv K_{S^{\prime}}+\sum b_{j} B_{j}+(1-a) E,
$$

with $a<\epsilon . S^{\prime}$ has Picard number 2, so it has two extremal rays, one of which is given by $E$. Contracting the other extremal ray we obtain a morphism $S^{\prime} \rightarrow S_{0}$ to a surface $S_{0}$. In fact if $S_{0}$ were a curve, by taking the intersection of $K_{S^{\prime}}+\sum b_{j} B_{j}+$ $(1-a) E$ with a general fiber, we have

$$
\sum n_{i} b_{j}+n(1-a)=2,
$$

where $n_{i} \geq 0$ and $n>0$ are integers, which contradicts Lemma 2.3, provided we choose $\epsilon=m f_{2}(\mathscr{A}, 2)$. Then $S_{0}$ is a log del Pezzo of Picard number 1 . Now $g$ does not contract $E$, and

$$
g_{*}\left(K_{S^{\prime}}+\sum b_{j} B_{j}+(1-a) E\right)=K_{S_{0}}+(1-a) E+\sum b_{j} g_{*}\left(B_{j}\right) \equiv 0 .
$$

Since $K_{S}+B \equiv 0$ we still have that $\left(S_{0},(1-a) E+\sum b_{j} g_{*}\left(B_{j}\right)\right)$ is a KLT pair. Then, applying Theorem 2.2 to $S_{0}$ with $b_{E}=1, x_{E}=(1-a)$, all other $x_{j}=1$ and $\epsilon=\delta$, we conclude that $K_{S_{0}}+(1-a) E+\sum b_{j} g_{*}\left(B_{j}\right)$ is not numerically equivalent to zero, which is a contradiction. 
Lemma 2.5. Let $(S, B) \rightarrow C$ be a morphism from a surface to a curve, such that the generic fiber is $\mathbb{P}^{1}$ and let $(S, B)$ be KLT with the coefficients of $B$ in a DCC set $\mathscr{A}$. Then $S$ is $\epsilon=\epsilon(\mathscr{A})$-log canonical.

Proof. Suppose that this is not the case. Then extract a divisor $f: S^{\prime} \rightarrow S$ such that

$$
f^{*}\left(K_{S}+\sum b_{j} B_{j}\right) \equiv K_{S^{\prime}}+\sum b_{j} B_{j}+(1-a) E,
$$

with $a<\epsilon$. Running a minimal model program for $\left(S^{\prime}, \sum b_{j} B_{j}\right)$ that does not contract $E$, we end up with a surface $S^{\prime \prime}$ that is either log del Pezzo or admits a Fano contraction to a curve. Furthermore the coefficients of $E$ in the pair $\left(S^{\prime \prime}, B^{\prime \prime}\right)$ is $(1-a)$ and $S^{\prime \prime}+B^{\prime \prime} \equiv 0$.

In the case $S^{\prime \prime}$ is log del Pezzo, we get a contradiction as in Lemma 2.4. If $S^{\prime \prime}$ admits a Fano contraction to a curve, by intersecting with a general fiber, we can apply the argument in Lemma 2.4 again.

Finally we deal with the case in which $K_{S}$ is numerically trivial.

Lemma 2.6. There is $\epsilon>0$ such that every KLT surface with $K_{S} \equiv 0$ is $\epsilon$-log canonical.

Proof. Set $\epsilon=\min \left\{\beta(\{1\}), m f_{2}\left(\{1\}^{\prime}, 2\right)\right\}$. Suppose that $S$ is not $\epsilon$-log canonical and extract a divisor $E$ by $f: S^{\prime} \rightarrow S$ such that $f^{*}\left(K_{S}\right)=K_{S^{\prime}}+(1-a) E$ and $0<a<\epsilon$. Then running a minimal model for $S^{\prime}$, which does not contract $E$, we can apply one of the above two cases.

\section{Bounding the index}

Observe that the weighted projective space $\mathbb{P}(a, b, c)$ with three lines $\left(x_{i}=0\right)$ forms an unbounded family of lc surface pairs when we vary $a, b$ and $c$, and the coefficients are in the DCC set $\{1\}$. However by restricting to the KLT case we have the boundedness result:

Theorem 3.1. If $(S, B)$ is a KLT pair of dimension 2 such that $K_{S}+B \equiv 0$ and the coefficients of $B$ are in a DCC set $\mathscr{A}$, there is a natural number $b=b(\mathscr{A})$ such that $b\left(K_{S}+B\right)$ is Cartier and $H^{0}\left(S, b\left(K_{S}+B\right)\right)=1$.

From Theorem 2.1 we know that there is an $\epsilon>0$ such that $(S, B)$ is $\epsilon-\log$ canonical; we will assume $0<\epsilon<1 / \sqrt{3}$.

Lemma 3.2 [Alexeev and Mori 2004, Lemma 1.2, Theorem 1.8]. Let X be a nonsingular projective surface and $B=\sum b_{j} B_{j}$ be an $\mathbb{R}$-divisor on $X$ with $0 \leq b_{j} \leq$ $1-\epsilon<1$. Assume $K_{X}+B \equiv 0$. Then

(1) if $E$ is an irreducible curve on $X$ and $E^{2}<0$, then $E \cong \mathbb{P}^{1}$ and $E^{2}>-2 / \epsilon$;

(2) $\rho(X) \leq 128 / \epsilon^{5}$. 
Proposition 3.3. Let $(S, B)$ be as in Theorem 3.1. There exists an integer $t=t(\epsilon)$ such that for any Weil divisor $D$ on $S, t D$ is Cartier.

Proof. The argument is parallel to the one in [Alexeev and Mori 2004, Lemma 3.7], though there $S$ is assumed to be a del Pezzo surface of Picard number 1.

Take the minimal resolution $f: \tilde{S} \rightarrow S$ of $S$ and write

$$
f^{*}\left(K_{S}\right)=K_{\tilde{S}}+\sum a_{i} E_{i} .
$$

Applying Lemma 3.2, we conclude that the determinant $t$ of the intersection matrix of the exceptional curves $\left(-F_{i} F_{k}\right)_{i k}$ is bounded by

$$
t \leq[2 / \epsilon]^{\left[128 / \epsilon^{5}\right]} \text {. }
$$

Since $S$ has rational singularities, for any Weil divisor $D$ on $S, t D$ is a Cartier divisor.

In the next proposition we repeatedly use the trivial property of DCC set stated in the following Lemma.

Lemma 3.4. Let $c$ be a positive real number and $\mathscr{A} \subset[0,1]$ a DCC set. Then there are a finite number of ways to write $c$ as the sum of $a_{i} \in \mathcal{A}$.

Proposition 3.5. Let $(S, B)$ be as in Theorem 3.1. There exists an integer $t=t(\epsilon)$ such that $t\left(K_{S}+B\right)$ is Cartier.

Proof. This follows from Proposition 3.3 once we make sure that there is a uniform multiple of $K_{S}+B$ that is a Weil divisor for all pairs $(S<B)$ satisfying our conditions.

Running a minimal model program for $S$, we end up with a KLT surface $f$ : $S \rightarrow S^{\prime}$. If $t\left(K_{S}^{\prime}+B^{\prime}\right)$ is Cartier, since

$$
f^{*}\left(t\left(K_{S^{\prime}}+B^{\prime}\right)\right) \sim t\left(K_{S}+f_{*}^{-1} B^{\prime}+a_{i} E_{i}\right)
$$

and

$$
K_{S}+f_{*}^{-1} B^{\prime}+a_{i} E_{i} \equiv K_{S}+B \equiv 0,
$$

we conclude that $K_{S}+f_{*}^{-1} B^{\prime}+a_{i} E_{i}=K_{S}+B$, so it suffices to prove it for $\left(S^{\prime}, B^{\prime}\right)$. $S^{\prime}$ is either of Kodaira dimension 0 , has a contraction to a curve with general fiber $\mathbb{P}^{1}$ or a log del Pezzo surface, so we need only prove the proposition in these cases.

Case 1: $S$ has Kodaira dimension 0 . Then $B=0$ and it follows directly from Proposition 3.3.

Case 2: $S$ admits a contraction to a curve $f: S \rightarrow C$ with the general fiber $\mathbb{P}^{1}$. By taking the intersection of $(S, B)$ with a generic fiber, we have

$$
2=\sum_{f\left(B_{j}\right) \neq \mathrm{pt}} a_{j}, \quad \text { where } a_{j} \in \mathscr{A}^{\prime} .
$$


On the other hand, applying the canonical bundle formula in this simple case, we have

$$
K_{S}+B \equiv f^{*}\left(K_{C}+B_{C}\right) \equiv 0, \quad \text { where } B_{C}=\sum_{f\left(B_{j}\right)=P_{j}} \frac{a_{j}+n-1}{n} P_{j} .
$$

From Lemma 3.4 we know that there are only finitely many choices of $a_{j}$, and we choose a natural number $N=N(\mathscr{A})$ that clears all the denominators of $a_{j}$.

Case 3: $S$ is a log del Pezzo surface of Picard number one. For $t$ as in Proposition $3.3, K_{S}+\sum b_{j} B_{j} \equiv 0$ implies that

$$
-t K_{S}^{2}=\sum t b_{j} B_{j} \cdot K_{S}
$$

Here $t K_{S}^{2}$ and $t B_{j} \cdot K_{S}$ are integers. From the proof of [Alexeev and Mori 2004, Lemma 3.7], we can bound $t K_{S}^{2}$ by

$$
t K_{S}^{2} \leq[2 / \epsilon]^{\left[128 / \epsilon^{5}\right]}([2 / \epsilon]+2)^{2} .
$$

Lemma 3.4 implies that there are only finitely many possible $b_{i}$ appearing as the coefficients of the boundary divisor. So there is a positive number $N=N(\mathscr{A})$ such that $N K_{S}$ and $N\left(b_{j} B_{j}\right)$ are all Cartier divisors.

In view of this discussion, if we take the minimal resolution $\pi: \tilde{S} \rightarrow S$ of $S$ and pull back $K_{S}+B$, then the denominators can be killed by a uniform multiple $t=t(\epsilon)$. Running a minimal model program for $\tilde{S}$, thus we only need to prove Theorem 3.1 in the case that $S$ is a smooth minimal surface and that the coefficients of $B$ all have the form $r / t$ for some uniform $t$. When $K_{S} \equiv 0$, then $B=0$, then it is from the classification theory of smooth surfaces; when $S$ is rational, a Cartier divisor is numerically trivial if and only if it is a trivial divisor. Thus the only tricky case is the following.

Lemma 3.6. If $S$ is a smooth minimal ruled surface over a curve of positive genus and there is $a \mathbb{Q}$-divisor $B$ such that $(S, B)$ is $K L T$ and $K_{S}+B \equiv 0$, then $S=E \times \mathbb{P}^{1}$, where $E$ is an elliptic curve. In particular, if there is some integer $N$ such that $N B$ is an integer divisor, then $\mathrm{O}\left(N\left(K_{S}+B\right)\right) \cong O_{S}$.

Proof. We use conventions for ruled surfaces as in [Hartshorne 1977, V.2]. Let $\pi: S \rightarrow C$ be $\mathbb{P}(\mathscr{E})$ over a curve $C$ of positive genus $g$ and let the general fiber of $\pi$ be $F$. We can assume $H^{0}(\mathscr{E}) \neq 0$ and $H^{0}(\mathscr{E} \otimes L)=0$ for any line bundle $L$ of degree -1 . Then $e=-\operatorname{deg}(\mathscr{E})$ is an invariant of $S$. There is a section $C_{0}$ such that $\left.C_{0}\right|_{C_{0}}=\bigwedge^{2} \mathscr{E}$, which we denote as $D$. Then $\operatorname{deg}(D)=-e$.

$$
K_{S} \sim-2 C_{0}+\pi^{*}\left(K_{C}+D\right) .
$$


Then we have

$$
0=\left(K_{S}+B\right) \cdot C_{0}=e+2 g-2+t C_{0}^{2}+\sum_{B_{i} \neq C_{0}} b_{i} B_{i} \cdot C_{0} \geq 2 g-2+(1-t) e,
$$

where $t<1$ is the coefficient of $C_{0}$ in $B$. So $e \leq 0$, and if $e=0$, then $g=1$. On the other hand, if $e<0$, it follows from [Hartshorne 1977, V.2.21] that

$$
B \cdot C_{0} \geq\left(2 C_{0}+e F\right) \cdot C_{0} \geq-e,
$$

so again we conclude $g=1$. And the equality holds only when for each irreducible component $B_{i}$ of $\operatorname{Supp}(B), g_{i}: B_{i} \rightarrow C$ is unramified.

Now we have $B_{i} \equiv a\left(2 C_{0}+e F\right)$ and $B_{i}^{2}=0$.

Claim. For $g: B_{i} \rightarrow C$, there are line bundles $M$ and $N$ on $B_{i}$ such that

$$
0 \rightarrow \mathscr{O} \rightarrow g^{*}(\mathscr{E}) \otimes M \rightarrow N \rightarrow 0 \quad \text { and } \quad \operatorname{deg} N=0 .
$$

Proof of the claim. It suffices to show that $h\left(B_{i}\right)^{2}=0$, where $h: B_{i} \rightarrow \mathbb{P}_{B_{i}}\left(g_{i}^{* \mathscr{E}}\right)$ is the induced section. But for $B_{i} \subset \mathbb{P}(\mathscr{E})$,

$$
0 \rightarrow I_{B_{i}} / I_{B_{i}}^{2} \rightarrow \Omega_{\mathbb{P}(\mathscr{E})}^{1} \otimes B_{i} \rightarrow \Omega_{B_{i}}^{1} \rightarrow 0
$$

where $\operatorname{deg}\left(I_{B_{i}} / I_{B_{i}}^{2}\right)=0$. Since $B_{i} \rightarrow C$ is unramified, the same exact sequence indeed also computes the conormal bundle of $h\left(B_{i}\right) \subset \mathbb{P}_{B_{i}}\left(g_{i}^{* \mathscr{E})}\right.$; thus we conclude that $h\left(B_{i}\right)^{2}=0$.

If $e>0$, then $\mathscr{E}$ is indecomposable which also means it is stable. But the exact sequence of the claim shows that $g^{*}(E)$ is not semistable, which is a contradiction.

When $e=0$, if $\mathscr{E}$ is indecomposable, then $\mathscr{E}$ is given by a nonsplit extension

$$
0 \rightarrow \mathfrak{O} \rightarrow \mathscr{E} \rightarrow \mathbb{O} \rightarrow 0
$$

For any isogeny $g: B \rightarrow C$, if there is a surjection $g^{*}(\mathscr{E}) \rightarrow G \rightarrow 0$ for a line bundle $G$ of degree 0 , then it is given by the pull-back of the above exact sequence. So $B=2 C_{0}$, which contradicts the assumption that $(S, B)$ is KLT. Similarly, if $\mathscr{E}=0 \oplus L$ for some degree-0 bundle $L$, we have at most two candidates for $B_{i}$, which again contradicts $(S, B)$ being KLT.

Finally, when $\mathscr{E}=\mathscr{O}^{2}$, all candidates of $B_{i}$ are proportional to $C_{0}$. Thus the last statement of the lemma holds.

\section{Effectiveness of the Iitaka fibration}

In this section we prove the effectiveness of the log Iitaka fibration for $(X, \Delta)$ a KLT pair of dimension three and four and log Kodaira codimension two. 
Consider a $\log$ resolution $\pi: X^{\prime} \longrightarrow X$ of $(X, \Delta)$ and write

$$
\pi^{*}\left(K_{X}+\Delta\right) \equiv K_{X^{\prime}}+\left(\pi^{-1}\right)_{*} \Delta+\sum_{i} e_{i} E_{i}
$$

with $E_{i}$ exceptional. There is a natural number $n$ such that $e_{i}<1-\frac{1}{n}$ for every $i$. Define

$$
\Delta^{\prime}=\left(\pi^{-1}\right)_{*} \Delta+\sum\left(1-\frac{1}{n}\right) E_{i} .
$$

Since all $E_{i}$ are exceptional divisors, we have

$$
H^{0}\left(X^{\prime},\left\lfloor m\left(K_{X^{\prime}}+\Delta^{\prime}\right)\right\rfloor\right)=H^{0}\left(X,\left\lfloor m\left(K_{X}+\Delta\right)\right\rfloor\right) .
$$

By replacing the $\mathscr{A}$ with the DCC set $\mathscr{A} \cup\left\{1-\frac{1}{n} \mid n \in \mathbb{N}\right\}$, we can assume that $X$ is smooth and $\Delta$ is simple normal crossing. Furthermore, we can assume that there is a morphism $f: X \longrightarrow Y$ giving the Iitaka fibration for $K_{X}+\Delta$ with $Y$ a smooth projective variety of dimension $m$ [Lazarsfeld 2004, 2.1.C]. For $F$ the general fiber of $f$, we have that $\kappa\left(K_{F}+\Delta_{F}\right)=0$.

From Kawamata's canonical bundle formula, we know that there is a $\mathbb{Q}$-line bundle $M=M_{(M, \Delta) / Y}^{S S}$, a $\mathbb{Q}$-divisor $B$ on $Y$ and a $\mathbb{Q}$-divisor $R$ on $X$ such that

$$
K_{X}+\Delta \sim_{\mathbb{Q}} f^{*}\left(K_{Y}+B+M+B\right)+R,
$$

where the terms in the formula satisfy the property that if $b\left(K_{F}+\Delta_{F}\right)$ is Cartier and $h^{0}\left(F, b\left(K_{F}+\Delta_{F}\right)\right)=1$, then for any $r \in \mathbb{Z}_{\geq 0}$ divided by $b$,

$$
H^{0}\left(X,\left\lfloor r\left(K_{X}+\Delta\right)\right\rfloor\right)=H^{0}\left(Y,\left\lfloor r\left(K_{Y}+M+B\right)\right\rfloor\right),
$$

[Fujino and Mori 2000, Theorem 4.5]. $M$ and $B$ are usually referred as the moduli part and the boundary part of the algebraic fiber space.

Proof of Theorem 1.3. First we can assume that $\left(F, \Delta_{F}\right)$ is a minimal pair, that is, $K_{F}+\Delta_{F} \equiv 0$. In fact, from [Kollár 2007, Definition 8.4.6], we know the moduli part $M$ only depends on the birational class of the morphism $X \rightarrow Y$, and we can choose one such that the generic fiber is minimal. Hence Theorem 2.1 implies that $\left(F, \Delta_{F}\right)$ is $\epsilon=\epsilon(\mathscr{A})$-lc.

Let $d$ be the smallest positive integer such that $d\left(K_{F}+\Delta_{F}\right)$ is Cartier and $h^{0}\left(F, d\left(K_{F}+\Delta_{F}\right)\right)=1$. Note that here we do not need the uniform integer from Theorem 1.3. Then we can construct the corresponding cyclic cover $g: E \rightarrow F$, and let $\phi: E^{\prime} \rightarrow E$ be the minimal resolution of $E$. Then, as in [Fujino and Mori 2000, Theorem 3.1], it suffices to bound the second Betti number of $E^{\prime}$. Write $g^{*}\left(K_{F}+\Delta_{F}\right)=K_{E}+\Delta_{E}$. From [Kollár and Mori 1998, 5.20.3], we know $\left(E, \Delta_{E}\right)$ is $\epsilon$-log canonical, provided that so is $\left(F, \Delta_{F}\right)$, which is the case because of Theorem 2.1. Pulling back to $E^{\prime}$, we conclude that $\left(E^{\prime}, \Delta_{E^{\prime}}\right)$ is $\epsilon$-log canonical. 
Then [Alexeev and Mori 2004, Theorem 1.8] implies that $\rho\left(E^{\prime}\right) \leq 128 / \epsilon^{5}$. Thus the second Betti number of $E^{\prime}$ is bounded by a constant depending only on $\mathscr{A}$.

The remainder of the proof was indeed given in [Todorov 2008, Section 3]. We give a sketch here. First we assume that the generic fiber $\left(F, \Delta_{F}\right)$ is $\log$ smooth. Then, as in [Kollár 2007, 8.4.5(7)], we can define a local system $\mathbb{V}$ on an open set $U \subset X$. Furthermore, if the monodromy action on $R^{2} f_{*}(\mathbb{V})$ is unipotent, the moduli part $M$ is identified with the bottom Hodge filtration of $R^{2} f_{*}(\mathbb{V})$, which is an integral divisor. In general, we take a $\log$ resolution $\tilde{F} \rightarrow F$ and write

$$
\pi^{*}\left(K_{F}+\Delta_{F}\right)=K_{\tilde{F}}+\Delta_{\tilde{F}}-G,
$$

where $\left\lfloor\Delta_{\tilde{F}}\left\lfloor=0, G \geq 0\right.\right.$ is an integral divisor and $\operatorname{Supp}\left(\Delta_{\tilde{F}}\right)$ has no common component with $G$. Near every codimension one point $P$, there is a Galois cover $Y^{\prime}$ of $Y$ such that when we pull back everything to $Y^{\prime}$, the monodromy action becomes unipotent near $P$. Thus as in [Fujino and Mori 2000, 3.6], the remaining question is to bound the character of $G \rightarrow \mathbb{Y}^{*}$ corresponding to the representation of $G$ on $H^{0}(\tilde{F}, \mathcal{O}(G))$. If we write $\tilde{E}$ as the degree- $b$ cyclic cover corresponding to $b\left(K_{\tilde{F}}+\Delta_{\tilde{F}}-G\right) \sim 0$, then $H^{0}(\tilde{F}, \mathcal{O}(G))$ is a direct summand of the bottom piece of the Hodge filtration of $H^{2}(\tilde{E}, \mathbb{C})$, which is also the bottom piece of $H^{2}\left(E^{\prime}, \mathbb{C}\right)$ since $\tilde{E}$ and $E^{\prime}$ are birational.

Then from [Fujino and Mori 2000, 3.8], we conclude that if the index of $\left(F, \Delta_{F}\right)$ is bounded by $b=b(\mathscr{A})$ and the second Betti number of the cyclic cover is bounded by $B=B(\mathscr{A})$, then the denominator of the moduli part is bounded by

$$
a=b \cdot \operatorname{lcm}\left\{m \in \mathbb{Z}_{>0} \mid \varphi(m) \leq B\right\},
$$

where $\varphi$ denotes the Euler $\varphi$-function.

Theorem 4.1. Let the notation be as above. Assume that the coefficients of $\Delta$ are in a DCC set of rational numbers $A \subset[0,1]$. Let the dimension of $X$ be either three or four and the dimension of the general fiber be two. Then there is a constant $N$ depending only on the set $\mathscr{A}$ such that $\left\lfloor N\left(K_{X}+\Delta\right)\right\rfloor$ induces the Iitaka fibration.

We will prove this statement when the dimension of $X$ is four, and leave the easier dimension-three case to the reader. In fact, this follows directly from the argument in [Fujino and Mori 2000, Section 6], and Theorem 1.3.

As we mentioned before, the main theorem of [Viehweg and Zhang 2007] says that for an arbitrary dimensional smooth variety $X$ of Kodaira dimension 2, if we assume that the generic fiber of the Iitaka fibration is a smooth variety $F$, then there is a constant $N$ depending on the middle Betti number $b_{m}$ of $F$ and the index of $F$, such that $\left|N K_{X}\right|$ gives the Iitaka fibration. The following discussion shows this is still true for the log case. Then because of Theorems 3.1 and 1.3, where we prove that the analogous bounds for the Betti number and the index, which only 
depend on the DCC set $\mathscr{A}$, exist even in the log case, provided the generic fiber is of dimension 2, we in fact get unconditional bounds (depending only on coefficients set) as in Theorem 1.2.

Theorem 4.2. If $(W, D)$ is a KLT surface, $L$ is a nef $\mathbb{Q}$-divisor (not necessarily effective) such that and $K_{W}+D+L$ is big. Assume that a is a positive integer such that a $L$ is a Cartier divisor, the coefficients of $D$ are in a DCC set $\mathscr{B}$, then we have a uniform $N=N(a, \mathscr{B})$ such that $\left|\left\lfloor N\left(K_{W}+D+L\right)\right\rfloor\right|$ gives a birational map.

Proof of Theorem 4.1. We apply Theorem 4.2 to $W=C, D=B, L=M$. To check the assumptions, the coefficients of $B$ are of the form

$$
\frac{b+n-1}{n}, \quad \text { for some } b \in \mathscr{A} \cup\{0\} \text { and } n \in \mathbb{Z}_{>0},
$$

[Kollár 2007, Theorem 8.3.7(2)], which forms a DCC set depending on $\mathscr{A}$. And for $a$, it can be chosen as in the last part of the proof of Theorem 1.3.

Proof of Theorem 4.2. This is essentially proved in [Viehweg and Zhang 2007], so here we only give a sketch which streamlines the arguments by using tools in [Alexeev and Mori 2004]. As before, we can start by assuming that $W$ is smooth. The main observation is that in fact many of the results of Alexeev and Mori can be strengthened in such a way that instead of assuming $\left(W, D=\sum b_{j} D_{j}\right)\left(b_{j} \in \mathscr{B}\right)$ is big, we can assume that $(W, D+L)$ is big, where $L$ is a nef line bundle such that for any curve $C, L \cdot C$ is in another DCC set $\mathscr{b}$. Then we get the same conclusion by changing all our constants $c=c(\mathscr{B})$ to $c=c(\mathscr{B}, \mathscr{C})$. In particular:

Proposition 4.3. Let the notation be as in Theorem 4.2. Then there is a uniform $\beta=\beta(\Re, a)$ such that $K_{W}+\sum\left(1-x_{j}\right) b_{j} B_{j}+L$ is big, provided $x_{j} \leq \beta$.

Because of Proposition 4.3 and the fact that all positive $b_{j}$ have a lower bound, we can indeed assume that all $b_{j}$ are of the form $n_{j} / m$ for some $m=m(\mathscr{B}, a)$.

Then the usual argument of cutting log canonical centers [Todorov 2008] works as long as we can prove that the volume of $K_{W}+\sum\left(1-x_{j}\right) b_{j} B_{j}+L$ has a uniform lower bound.

Now we run the minimal model program, $f:(W, D) \rightarrow\left(W^{\prime}, D^{\prime}=f_{*}(D)\right)$, $L^{\prime}=f_{*} L$. Because $f^{*}\left(L^{\prime}\right) \geq L$, we have

$$
H^{0}\left(W,\left\lfloor n\left(K_{W}+D+L\right)\right\rfloor\right)=H^{0}\left(W^{\prime},\left\lfloor n\left(K_{W^{\prime}}+D^{\prime}+L^{\prime}\right)\right\rfloor\right) \text { for all } n \text {. }
$$

Case 1: $K_{W}+D$ is pseudo-effective. Then we end up with a minimal model $\left(W^{\prime}, D^{\prime}\right)$ such that $K_{W^{\prime}}+D^{\prime}$ is nef. If $K_{W^{\prime}}+D^{\prime}$ is big, this is so from [Alexeev and Mori 2004]. Otherwise,

$$
\left(K_{W^{\prime}}+D^{\prime}+L^{\prime}\right)^{2}=2\left(K_{W^{\prime}}+D^{\prime}\right) \cdot L^{\prime}+L^{\prime 2}>0,
$$

which has a uniform lower bound by our assumption. 
Case 2: $K_{W}+D$ is not pseudo-effective. Then we define the pseudo-effective threshold, which is the smallest number $e$ such that $K_{W}+D+e L$ is pseudoeffective. Note that since $K_{W}+D+L$ is big we have that $e \leq 1$. Then we have two subcases, for which we give a sketch of the argument. For details see the proof of [Viehweg and Zhang 2007, Proposition 2.7].

Case 2a: We end up with a log del Pezzo surface $\left(W^{\prime}, D^{\prime}\right)$ of Picard number 1 . So

$$
K_{W^{\prime}}+D^{\prime}+e L^{\prime} \equiv 0 .
$$

The discussion above about the generalization of [Alexeev and Mori 2004] indeed implies that $1-e$ has a uniform lower bound. So $\left(K_{W^{\prime}}+D^{\prime}+L^{\prime}\right)^{2}=(1-e) L^{\prime 2}$. Case $3 b$ : We end up with a Fano contraction $\left(W^{\prime}, D^{\prime}\right)$ to a curve. By the argument above, we can assume $L^{\prime}$ is not big. Then $W^{\prime}$ is of Picard number 2 and generated by the fiber of the Fano contraction and $L^{\prime}$. Taking the intersection of $K_{W}^{\prime}+D^{\prime}+e L^{\prime}$ with the fiber, we conclude that $e$ is uniformly far from 1 . Since the coefficients of $D^{\prime}$ have bounded denominators, the fact that $\left(K_{W^{\prime}}+D^{\prime}\right) \cdot L^{\prime}$ is positive actually implies it is uniformly away from 0 . So the volume

$$
\left(K_{W^{\prime}}+D^{\prime}+L^{\prime}\right)^{2}=(1-e)\left(K_{W^{\prime}}+D^{\prime}\right) \cdot L^{\prime}
$$

is bounded from below. This concludes all possible cases, and hence Theorem 4.2 is proved.

\section{Acknowledgment}

The authors thank Christopher Hacon, János Kollár and James $\mathbf{M}^{\mathrm{c}}$ Kernan for numerous conversations and suggestions. This material is partly based upon work carried out by the second author while at the Institute for Advanced Study and supported by the NSF under agreement DMS-0635607. Any opinions, findings and conclusions or recommendations expressed in this material are those of the author and do not necessarily reflect the views of the NSF.

\section{References}

[Alexeev 1994] V. Alexeev, "Boundedness and $K^{2}$ for log surfaces", Internat. J. Math. 5:6 (1994), 779-810. MR 95k:14048 Zbl 0838.14028

[Alexeev and Mori 2004] V. Alexeev and S. Mori, "Bounding singular surfaces of general type", pp. 143-174 in Algebra, arithmetic and geometry with applications (West Lafayette, IN, 2000), edited by C. Christensen et al., Springer, Berlin, 2004. MR 2005f:14077 Zbl 1103.14021

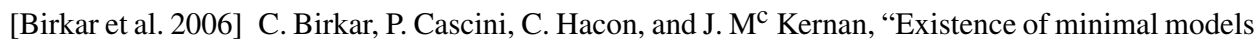
for varieties of log general type", preprint, 2006. arXiv 0610203

[Fujino and Mori 2000] O. Fujino and S. Mori, "A canonical bundle formula", J. Differential Geom. 56:1 (2000), 167-188. MR 2002h:14091 Zbl 1032.14014 
[Hacon and $\mathrm{M}^{\mathrm{c}}$ Kernan 2006] C. D. Hacon and J. $\mathrm{M}^{\mathrm{c}}$ Kernan, "Boundedness of pluricanonical maps of varieties of general type", Invent. Math. 166:1 (2006), 1-25. MR 2007e:14022 Zbl 1121.14011

[Hartshorne 1977] R. Hartshorne, Algebraic geometry, Graduate Texts in Mathematics 52, Springer, New York, 1977. MR 57 \#3116 Zbl 0367.14001

[Kawamata 1986] Y. Kawamata, "On the plurigenera of minimal algebraic 3-folds with $K \approx 0$ ", Math. Ann. 275:4 (1986), 539-546. MR 88c:14049 Zbl 0582.14015

[Kawamata 1998] Y. Kawamata, "Subadjunction of log canonical divisors, II", Amer. J. Math. 120:5 (1998), 893-899. MR 2000d:14020 Zbl 0919.14003

[Kollár 1994] J. Kollár, "Log surfaces of general type; some conjectures", pp. 261-275 in Classification of algebraic varieties (L'Aquila, 1992), edited by C. Ciliberto et al., Contemp. Math. 162, Amer. Math. Soc., Providence, RI, 1994. MR 95c:14042 Zbl 0860.14014

[Kollár 2007] J. Kollár, "Kodaira's canonical bundle formula and adjunction”, pp. 134-162 in Flips for 3-folds and 4-folds, edited by A. Corti, Oxford Lecture Ser. Math. Appl. 35, Oxford Univ. Press, Oxford, 2007. MR MR2359346

[Kollár and Mori 1998] J. Kollár and S. Mori, Birational geometry of algebraic varieties, Cambridge Tracts in Math. 134, Cambridge Univ. Press, New York, 1998. MR 2000b:14018 Zbl 0926.14003

[Lazarsfeld 2004] R. Lazarsfeld, Positivity in algebraic geometry, I, Ergebnisse der Math. (3) 48, Springer, Berlin, 2004. MR 2005k:14001a Zbl 1093.14501

[Pacienza 2007] G. Pacienza, "On the uniformity of the Iitaka fibration", preprint, 2007. To appear in Math. Research Letters. arXiv 0709.0310

[Prokhorov and Shokurov 2009] Y. Prokhorov and V. Shokurov, "Towards the second main theorem on complements", J. Algebraic Geom. 18 (2009), 151-199.

[Ringler 2007] A. Ringler, "On a conjecture of Hacon and McKernan in dimension three", preprint, 2007. arXiv 0708.3662

[Shokurov 1992] V. Shokurov, "3-fold log flips", Izv. Russ. A. N. Ser. Mat. 56:1 (1992), 105-203. In Russian; translated in Russ. Acad. Sci., Izv., Math. 40:1 (1993), 95-202. Zbl 0785.14023

[Takayama 2006] S. Takayama, "Pluricanonical systems on algebraic varieties of general type", Invent. Math 165:3 (2006), 551-587.

[Todorov 2008] G. Todorov, "Effective log Iitaka fibrations for surfaces and threefolds", preprint, 2008. arXiv 0805.3494

[Viehweg and Zhang 2007] E. Viehweg and D. Zhang, "Effective Iitaka fibrations", preprint, 2007. To appear in J. Alg. Geom. arXiv 0707.4287

Communicated by Shigefumi Mori

Received 2009-01-20 Revised 2009-07-09 Accepted 2009-08-17

gtodorov@math.princeton.edu Department of Mathematics, Princeton University, Princeton, NJ 08544, United States

cyxu@mit.edu

Department of Mathematics, Massachusetts Institute of Technology, 77 Massachusetts Avenue, Cambridge, MA 02139, United States 